OPEN

SUBJECT AREAS:

LIGHT HARVESTING

OPTICAL MATERIALS

Received

3 February 2014

Accepted

24 March 2014

Published

10 April 2014

Correspondence and requests for materials should be addressed to K.P.G. Ighiggino@ unimelb.edu.au) or W.W.H.W.

(wwhwong@unimelb. edu.au)

\section{Concentrating Aggregation-Induced Fluorescence in Planar Waveguides: A Proof-of-Principle}

\author{
James L. Banal, Jonathan M. White, Kenneth P. Ghiggino \& Wallace W. H. Wong
}

Bio2 1 Institute, School of Chemistry, The University of Melbourne, Parkville 3010, Australia

The photophysical properties of fluorescent dyes are key determinants in the performance of luminescent solar concentrators (LSCs). First-generation dyes - coumarin, perylenes, and rhodamines - used in LSCs suffer from both concentration quenching in the solid-state and small Stokes shifts which limit the current LSC efficiencies to below theoretical limits. Here we show that fluorophores that exhibit aggregation-induced emission (AIE) are promising materials for LSC applications. Experiments and Monte Carlo simulations show that the optical quantum efficiencies of LSCs with AIE fluorophores are at least comparable to those of LSCs with first-generation dyes as the active materials even without the use of any optical accessories to enhance the trapping efficiency of the LSCs. Our results demonstrate that AIE fluorophores can potentially solve some key limiting properties of first-generation LSC dyes.

uminescent solar concentrators (LSCs) rely on the absorption of solar light by highly luminescent materials embedded in glass or plastic substrates (Figure 1). Subsequent waveguiding of emission to the thin edges of the substrate concentrates the luminescence which can, in principle, be used to improve the output of photovoltaic devices. The simple device configuration of LSCs means photovoltaics can be integrated into urban environments, such as windows and walls, at low cost ${ }^{1}$. Currently, factors limiting LSC efficiency include reduced fluorescence quantum yield in the solid state arising from dye aggregation ${ }^{2}$, reabsorption of dye emission (i.e. due to a small Stokes shift), and limited trapping efficiencies for conventional glass or plastic waveguides. Commercial laser dyes such as rhodamines, coumarins, and perylenes - first-generation dyes - have been used for LSCs. A common feature of such dyes is their highly planar conjugated structure, which is conducive to the formation of non-emissive aggregates particularly at the high concentration required for total light absorption. Consequently, the fluorescence quantum yield of these dyes in the solid state can be lower than that measured in solution. Dilute concentrations of the chromophores can be used but at the cost of lower light absorption. These dyes also have significant overlap between their absorption and emission spectrum resulting in re-absorption losses ${ }^{2}$. Secondgeneration dyes based on quantum dots or semiconductor nanorods $s^{3,4}$, inorganic phosphors ${ }^{5}$, and chromophores in light-emitting diodes ${ }^{6}$ are promising materials for LSCs due to their large Stokes shifts which result in lower reabsorption but they generally exhibit reduced fluorescence quantum yields compared to first-generation dyes. These materials also suffer from concentration quenching effects, which limit the concentration of the dyes that can be used to achieve high film absorption. Other LSC losses arising from limited trapping efficiencies can be addressed by using higher refractive index materials, wavelength-selective mirrors, or homeotropically aligned molecules on the waveguide ${ }^{1}$.

Recent molecules designed to address the aggregate fluorescence quenching and reabsorption issues simultaneously have only been partially successful, i.e. while energy transfer in multichromophoric dendrimers can increase the Stokes shift, the fluorescence quantum yields of these chromophores in the solid-state are low ${ }^{7,8}$. In addition, these chromophores are often macromolecules that require multi-step synthesis which are not ideal for industrial scale-up. Baldo and co-workers have demonstrated that energy transfer strategies in non-covalent chromophore mixtures are also effective in increasing the Stokes shift and reducing the effects of concentration quenching by low-concentration doping in hosts similar to organic light-emitting diodes ${ }^{6,9}$. Molecules that meet all requirements - lossless solid-state fluorescence, negligible re-absorption, and low synthetic cost - remain a key objective to improve LSC performance.

Fluorophores that exhibit aggregation induced emission have been used in biological applications ${ }^{10}$ and light emitting devices ${ }^{11}$. A characteristic of this class of fluorophores is emission that is greatly enhanced in the solid state compared to solution. This fluorescence behavior is widely referred to as aggregation induced emission 
(a)

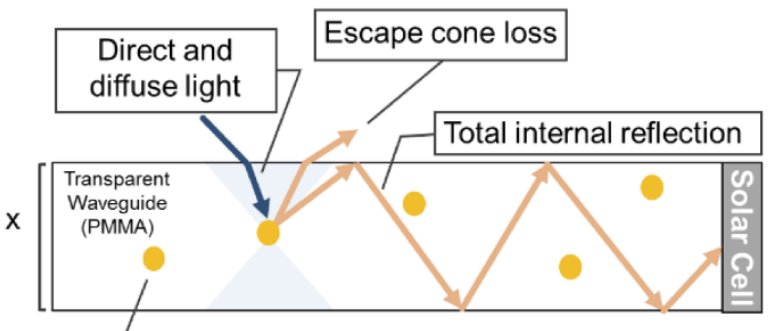

Chromophore

(b)

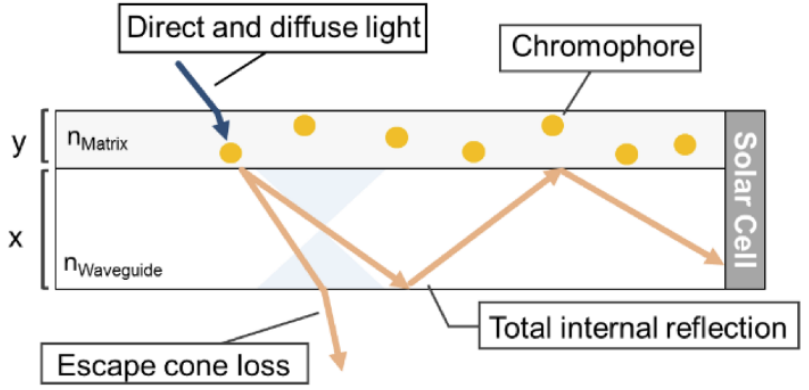

Figure $1 \mid$ Conventional structures for LSC applications: (a) millimeterthick (x) waveguides (typically polymer matrices) infused with fluorescent dyes and (b) micron-thick thin layer matrices (y) cast on top of a waveguide (typically glass). In this study, structure (b) is used with a neat film layer. $(\mathrm{n}=$ refractive index $)$.

(AIE) $)^{12}$. Enhancement of chromophore emission is not limited to aggregation in thin-films but also when dispersed in a polymer matrix or in frozen solution. It has been proposed that these conditions reduce non-radiative decay pathways arising from intramolecular rotation and channel the excitation energy towards fluorescence ${ }^{13}$.

Ray-tracing calculations have been successful in predicting the performance of LSCs and are particularly useful in understanding the optical losses that limit LSC performance ${ }^{2,14-16}$. Hence, ray-tracing models can be used as a screening method for potential chromophores for LSCs by using the spectroscopic properties of the chromophores as inputs for the model, i.e. extinction coefficient in solution; fluorescence spectra and quantum yield in the solid-state. In this letter, we disclose the use of a class of fluorophores that combine the three desirable properties mentioned previously in a LSC configuration using both modelling and experimental approaches. The use of AIE fluorophores mitigates reabsorption and solid-state quenching. To our knowledge this is the first demonstration of the application of AIE fluorophores for solar harvesting in a LSC configuration.

\section{Discussion}

A monolithic LSC was fabricated by drop casting $10 \% \mathrm{w} / \mathrm{w}$ of tetraphenylethene 1 (for structure see Figure 2a) in PMMA (the casting solution is $1 \% \mathrm{w} / \mathrm{w}$ total solids in chloroform) onto the surface of $10 \mathrm{~mm} \times 10 \mathrm{~mm} \times 1 \mathrm{~mm}$ glass substrates (geometric ratio $\mathrm{G}=$ 2.5). The size of the representative LSC is kept at such dimensions to fit the integrating sphere sample holder (Horiba Jobin Yvon integrating sphere accessory). The thickness of the PMMA film was measured using a stylus profilometer (Dektak 150 Stylus Profiler, Veeco USA) and was found to be $116 \mathrm{~nm}$. The chromophore concentration was limited to $10 \% \mathrm{w} / \mathrm{w}$ as higher concentrations led to inhomogeneous film quality. The LSC geometry is illustrated in Figure 1b. The excitation and emission spectra as well as the edge and face emission spectral profiles of the LSC are shown in Figures 2a and 2b, respectively. For both the modelling and experiment, the wavelength of maximum absorbance was used as the excitation wavelength
(315 nm). The absolute fluorescence quantum yield of the films was measured to be 0.4 using methods described previously ${ }^{2}$. The validity of the ray-tracing model to adequately describe the geometric optics of the LSC was verified by comparing the simulated and experimental light trapping efficiencies (Figure 2c).

We measured the optical edge efficiency $\left(\eta_{\text {edge }}\right)$ of the LSC defined as the ratio of the number of fluorescence photons that are waveguided to the edges relative to the number of the incident photons. Edge emissions were differentiated from face emissions using a black matte paint to mask edge emissions and reduce edge reflections. Optical edge efficiency takes into account the photophysical properties of the fluorophores in the LSC - quantum yield, absorption efficiency, Stokes shift, and re-absorption - in addition to the light trapping efficiency (Table 1$)^{6}$. The $\eta_{\text {edge }}$ of the LSC with tetraphenylethene in PMMA on a $10 \mathrm{~mm} \times 10 \mathrm{~mm} \times 1 \mathrm{~mm}$ glass plate measured in an integrating sphere (both with $G=2.5$ ) were found to be $13.2 \%$, which is close to the predicted value of $13.4 \%$ by simulation with the same geometric ratio and zero reabsorption loss. Ideally, an LSC should absorb most of the incident light to be efficient in the downconversion process. The LSC based on 1 in PMMA does not totally absorb the incident light, which limits the achievable efficiency of the LSC.

We further explored potential strategies to improve the performance of the LSC based on 1 using ray-trace modelling. The predicted $\eta_{\text {edge }}$ efficiency at $\mathrm{G}=50$ (assuming the same polymer film thickness, substrate thickness, and absorbance) is $13.7 \%$ with a negligible $0.2 \%$ reabsorption loss. Increasing the absorbance at $\mathrm{G}=50$ to a value of 2, the model predicts a modest increase in $\eta_{\text {edge }}$ to $25.1 \%$ with a predicted $0.2 \%$ reabsorption loss. In practice increasing the tetraphenylethene concentration to higher concentrations is limited due to increased light scattering losses, likely due to the formation of organic aggregates and microcrystals ${ }^{17}$. The $\eta_{\text {edge }}$ of the highest geometric ratio $(G=250)$ that can be calculated within reasonable computational cost (same film thickness, LSC thickness, and absorbance of 2.0 ) is $26.7 \%$ with a reabsorption loss of $0.3 \%$. The extinction coefficient $\left(\mathrm{ppm}^{-1} \mathrm{~cm}^{-1}\right)$ of $\mathbf{1}$ in the spectral region that overlaps with the emission spectrum is of the order of $10^{-5}$, consistent with the very low reabsorption losses.

While tetraphenylethene shows promising results in a LSC configuration, particularly in harvesting photons in the UV range, the emission range is not ideal for LSCs that are coupled with high efficiency silicon or GaAs photovoltaic cells. Extending the $\pi$-conjugation of the molecule should shift the absorption and emission to longer wavelengths but this could have adverse effects on its AIE behaviour ${ }^{18,19}$. For example extending the aromatic core might enhance intermolecular $\pi$ - $\pi$ interactions, forming non-emissive Jaggregates and resulting in quenching of the fluorescence. Hence, contorted aromatic fluorophores 2 to 4 (Figure 3) and fully substituted butadiene 5 were chosen as chromophores to extend the spectral absorption compared to tetraphenylethene. Synthetic procedures, absorption and emission spectra, and other spectroscopic information to verify that these compounds are AIE fluorophores are summarized in the Supporting Information. The crystal structures of chromophores 2 and 4 confirmed the contorted molecular structure (Figure 4). It was observed that chromophore 2 without the tert-butyl groups formed needle shaped crystals that exhibited selfwaveguiding of the emission to the crystal end faces under UV lamp irradiation (Figure 4c). This phenomenon might potentially be beneficial in luminescent solar concentrators based on crystalline microrods of these materials although this was not investigated further here.

The emission quantum yields for compounds 2 to 5 were measured as pristine films to verify that the compounds behave the same way as typical AIE compounds, i.e. fluorescent in the solid state. We have also investigated the AIE behavior of the compounds dissolved in glass-forming solvents at cryogenic temperatures, such as 
(a)

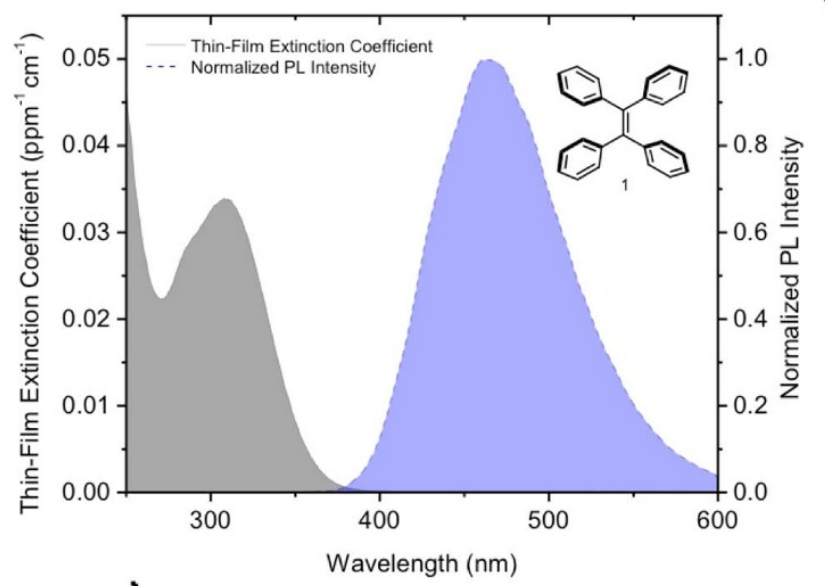

(c)

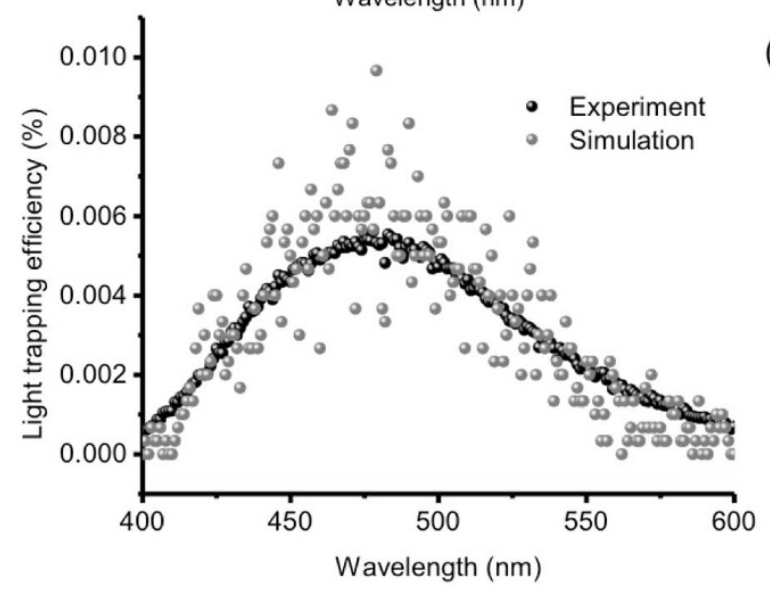

(b)

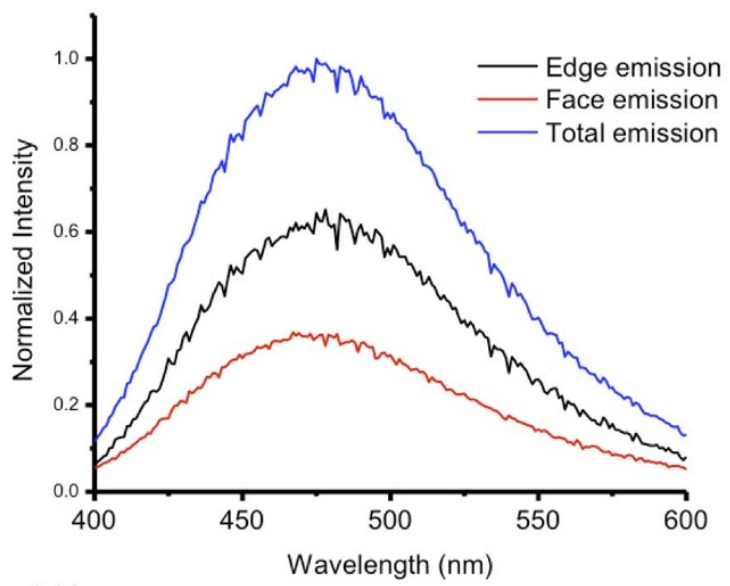

(d)

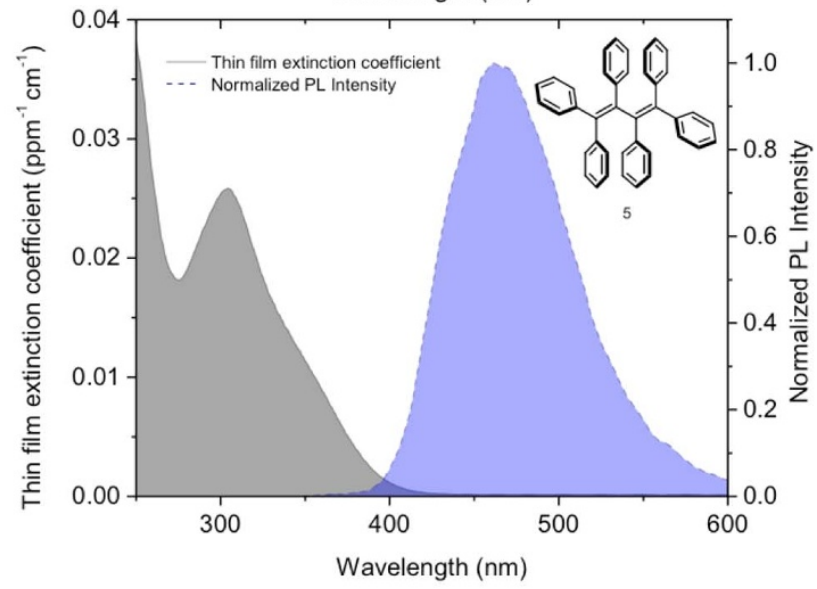

Figure $2 \mid$ (a) Extinction coefficient spectrum and normalized emission spectrum of $\mathbf{1}$ dispersed in PMMA as a thin-film. These spectra were used in the ray-tracing model. (b) Total, edge, and face emission spectra of the LSC. The edge and face emissions were resolved by blocking the edges of the LSC with a black matte paint with $0 \%$ transmission to minimize edge reflection. (c) Comparison of simulated and experimental light trapping efficiencies. Edge reflections were taken into account in the ray-tracing model. Light trapping efficiency $(\lambda)=\left[I_{\text {total }}(\lambda)-I_{\text {face }}(\lambda)\right] /\left[I_{\text {total }}(\lambda) d \lambda\right.$, where $I_{\text {total }}(\lambda)$ is the emission intensity of the whole LSC and $\mathrm{I}_{\text {face }}(\lambda)$ is the emission intensity when the edges of the LSC are blocked. Edges are blocked with black matte paint. Experimental total light trapping efficiency is $62.5 \%$ compared with simulated total light trapping efficiency of $65.2 \%$ (d) Thin-film absorption spectrum (in $\mathrm{ppm}^{-1} \mathrm{~cm}^{-1}$ units) and emission spectrum of 5 dispersed in PMMA on a $10 \mathrm{~mm} \times 10 \mathrm{~mm} \times 1 \mathrm{~mm}$ quartz substrate.

2-methyl tetrahydrofuran, and have found fluorescence behavior consistent with AIE compounds (see Figure S19). The pristine thin-film emission quantum yields of the fluorophores decrease with increasing number of the polyaromatic rings (2-4) albeit their structures are contorted and no evidence of $\pi-\pi$ interactions was apparent. The decreased fluorescence yields may be due to increased voids in the crystal lattice associated with the larger molecules, which would allow more mobility of the phenyl rings and favor

Table 1 | Spectroscopic properties and performance characteristics in a LSC of chromophores 1-5

\begin{tabular}{|c|c|c|c|c|c|c|c|}
\hline Chromophore & $\begin{array}{l}\text { Solution absorption } \\
\text { coefficient at } \lambda_{\max }{ }_{\text {[a] }} \\
\left(\times 10^{4} \mathrm{M}^{-1} \mathrm{~cm}^{-1}\right)\end{array}$ & $\begin{array}{c}\text { Thin film } \\
\text { absorption } \\
\lambda_{\text {max }}^{[b]}(\mathrm{nm})\end{array}$ & $\begin{array}{l}\text { Thin film fluorescence } \\
\qquad \lambda_{\text {max }}{ }^{[\mathrm{c}]}(\mathrm{nm})\end{array}$ & Quantum yield ${ }^{[d]}$ & $\begin{array}{l}\text { Stokes shift } \\
(\mathrm{eV})^{[\mathrm{e}]}\end{array}$ & $\begin{array}{c}\text { Experimental } \\
\eta_{\text {edge }} \text { at } G=2.5^{[f]}\end{array}$ & $\begin{array}{l}\text { Projected } \eta_{\text {edge }} \\
\text { at } G=50^{[g]}\end{array}$ \\
\hline 1 & 1.03 & 315 & 449 & $49.5 \%(40.8 \%)$ & 1.11 & $13.2 \%(13.4 \%)$ & $13.7 \%$ \\
\hline 2 & 1.90 & 314 & 493 & $9.9 \%$ & 1.43 & - & - \\
\hline 3 & 2.65 & 348 & 502 & $9.2 \%$ & 1.09 & - & - \\
\hline 4 & 6.42 & 301 & 455 & $4.1 \%$ & 1. & - & - \\
\hline 5 & 1.55 & 307 & 405 & $31.2 \%$ & 0.98 & - & $8.0 \%$ \\
\hline \multicolumn{8}{|c|}{ 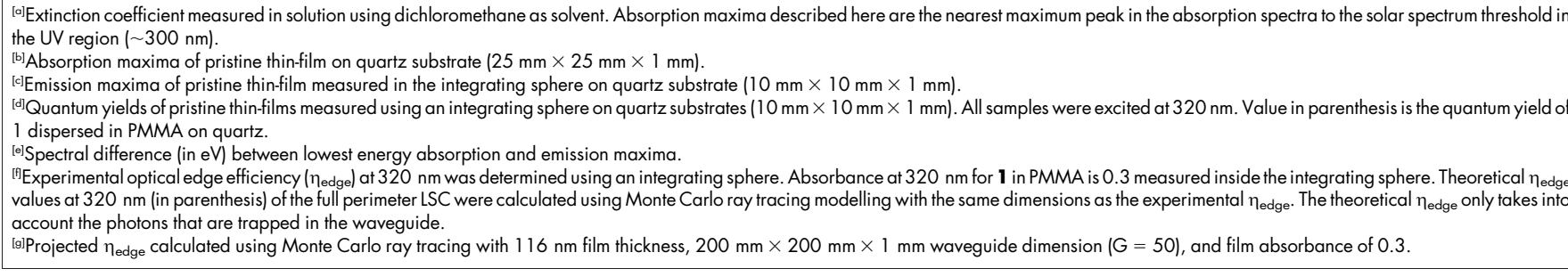 } \\
\hline
\end{tabular}


<smiles>CC(C)(C)c1ccc(C(c2ccccc2)=c2c3ccccc3c(=C(c3ccc(C(C)(C)C)cc3)c3ccc(C(C)(C)C)cc3)c3ccccc23)cc1</smiles>

Figure $3 \mid$ Structures of contorted and twisted polyaromatic hydrocarbons studied in this paper.

non-radiative decay. Extending the alkene length to butadiene (5), however, gave an AIE fluorophore with a quantum yield of $31 \%$ in the solid-state that is still lower compared to 1 . The increase in absorption range in $\mathbf{2}$ and $\mathbf{4}$ is minimal which suggests that there is very little or no effective conjugation that extended through the contorted aromatic rings. The large increase in absorption range for 3 relative to 1 is possibly due to the increase in the number of "ethene" bonds rather than an increase in the number of aromatic

(a)
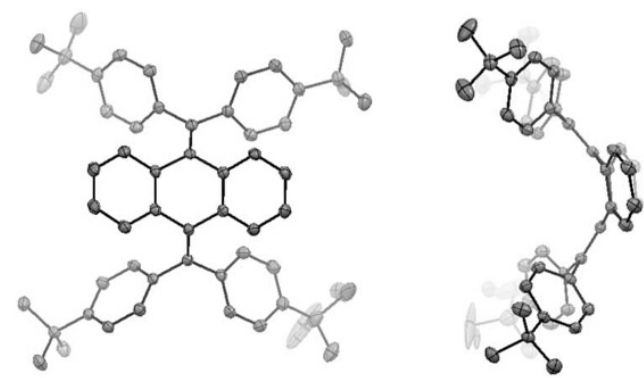

(b)
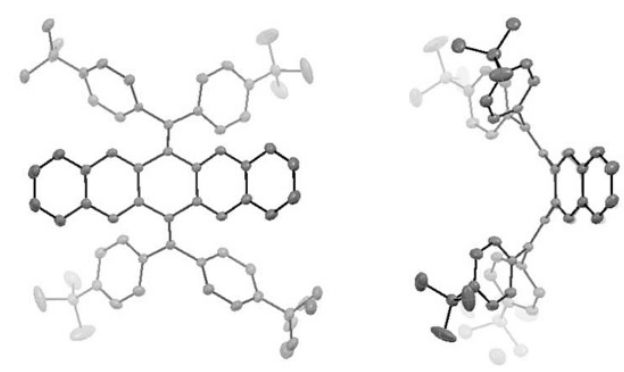

(c)
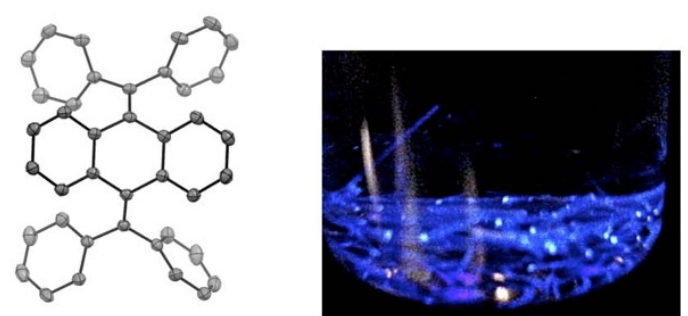

Figure 4 Crystal structures of compounds not previously reported - (a) Chromophore 2, (b) Chromophore 4, and (c) Crystal structure of chromophore 2 without ${ }^{t}$ Butyl groups and a photograph of crystals under UV light showing self-waveguiding of the luminescence. rings, which is also evident for 5 . The quantum yield of the fluorophores in solution cannot be measured reliably due to the very weak fluorescence from solutions (see Figure S19 for room temperature spectra).

The extent of spectral overlap between the absorption and emission spectrum and Stokes shift for $\mathbf{5}$ (Figure 2d) is comparable to $\mathbf{1}$ but the tail extinction coefficient that immediately overlaps with the emission spectrum is of the order of $10^{-3}$, two orders of magnitude higher than 1, which would suggest reabsorption losses will be more significant. Using the spectroscopic properties of 5 as model inputs, the projected $\eta_{\text {edge }}$ of $\mathbf{5}$ in PMMA for $\mathrm{G}=50$ assuming the same experimental film properties as 1 in PMMA (i.e. maximum film absorbance of 0.3 , film thickness of $116 \mathrm{~nm}$, and deposited on a glass substrate) is $8.0 \%$ (Table 1 ). The predicted reabsorption loss for an LSC based on $5(\mathrm{G}=50)$ is $2.3 \%$. Increasing the absorbance of the film of 5 to 2.0 while maintaining the same geometric ratio results an increase in $\eta_{\text {edge }}$ to $15.9 \%$ and an increase in reabsorption loss to $5.2 \%$. Further increasing the LSC dimensions to $\mathrm{G}=100$ results in a $\eta_{\text {edge }}$ of $14.8 \%$ with reabsorption contribution of $6.7 \%$. These results are consistent with the observation of Richards and co-workers that tail absorption is important in understanding reabsorption losses in $\mathrm{LSCs}^{20}$. The absorption spectra of thin-films must be carefully determined as the quality of the inputs in the ray-tracing model is critical in simulating LSC systems. As an example, the LSC described here could be used to improve the spectral sensitivity of a CdTe solar cell that has low absorption in the wavelength region where the LSC absorbs but is able to efficiently absorb the concentrated luminescence $^{21}$. Chromophores used in LSCs with emission wavelengths similar to the system here have been used for multicrystalline silicon solar cells ${ }^{22}$.

In summary, we have provided a proof-of-principle assessment of the merits of AIE fluorophores as potential emitters for LSCs using an established AIE fluorophore tetraphenylethene $\mathbf{1}$ as a baseline for the design of AIE fluorophores. Our results demonstrate that their unusually large Stokes shift leads to small reabsorption losses in large LSC dimensions. Losses due to concentration quenching are absent within this class of materials regardless of whether they are dispersed in polymer hosts or deposited as pristine films. We have also explored some design strategies to increase the absorption range of AIE molecules. While contorted polyaromatic rings may help prevent formation of non-emissive aggregates, their contribution towards extending the conjugation of an ethene core is less effective. Using heterocyclic rings might help extend the absorption and emission spectral range of these AIE molecules and studies towards this objective are in progress. 
11. Hong, Y., Lam, J. W. Y. \& Tang, B. Z. Aggregation-induced emission:

\section{Methods}

Characterization data and spectroscopic properties not shown can be found in the Supporting Information. All films were fabricated by the drop-casting method using a $1 \% \mathrm{w} / \mathrm{w}$ (total solids; $10 \% \mathrm{w} / \mathrm{w}$ of AIE material relative to PMMA) solution. The films were allowed to dry in room temperature for 24 hours before measurement. Emission quantum yields of all the chromophores were measured according to published procedures detailed elsewhere ${ }^{23}$. Photoluminescence of the chromophores for quantum yield measurements, optical edge efficiency, and light trapping efficiency were recorded using an integrating sphere accessory attached to a Horiba Jobin-Yvon FluoroLog-3. The spectra of all chromophores were corrected for detector response and background. Due to the sample holder configuration of the equipment, substrates used for measurement were limited to $10 \mathrm{~mm} \times 10 \mathrm{~mm} \times 1 \mathrm{~mm}$ dimensions. Quartz substrates were used for quantum yield measurements while glass was used in the optical edge efficiency measurement to match the refractive index of PMMA. Due to the small area of the quartz substrate for quantum yield measurement, films with thicknesses of the order of several $100 \mathrm{~nm}$ were required to produce homogenous films. All the films were directly excited and secondary excitations, i.e. transmitted light after incidence with the film that is absorbed again by the film after reflections in the integrating sphere, were accounted for as described previously ${ }^{23}$. Films for measuring the extinction coefficient were fabricated on a $25 \mathrm{~mm} \times 25 \mathrm{~mm} \times 1 \mathrm{~mm}$ quartz substrate. The reflectivity of the films, measured using an integrating sphere accessory attached to a UV/Vis spectrophotometer (Thermo Scientific Evolution 220 UV-Visible), was $2.5-3.0 \%$ from $250 \mathrm{~nm}$ to $750 \mathrm{~nm}$ for both 1 and 5 in PMMA. Film absorption for the ray-tracing model was then measured using a Cary $50 \mathrm{UV} / \mathrm{Vis}$ spectrophotometer, which was zero and blank corrected prior to measurement. Film thicknesses were measured using surface profilometry (Dektak 150 Stylus Profiler, Veeco USA) for the small LSCs and thin-film extinction measurements. The thickness of the films was adjusted to achieve an absorbance of 1.5 at $315 \mathrm{~nm}$ and $307 \mathrm{~nm}$ for 1 and 5 , respectively. Average film thicknesses for the thin-film extinction measurements (mean \pm sd; $\mathrm{n}=3$ ) for 1 and 5 in PMMA were $4900 \pm 200 \mathrm{~nm}$ and $4200 \pm$ $50 \mathrm{~nm}$, respectively. Each measurement $n$ was measured at different positions: middle, edge, and corner. Optical quantum efficiencies were measured with an integrating sphere as described by Baldo and co-workers ${ }^{24}$. The face and edge emissions were differentiated by blocking the edges with black matte paint (Abs $\sim 10$ from $300 \mathrm{~nm}-750 \mathrm{~nm})$

Projected values for light trapping efficiency and optical quantum efficiency were calculated using Monte Carlo ray tracing written in MATLAB developed previously ${ }^{2}$. Total input photon events are 100,000 in all cases. It should be noted that it is assumed that the emission of the chromophores is isotropic. Refractive indices used for the PMMA film and the glass waveguide were 1.49 and 1.53 respectively. A schematic diagram of the photon events in the ray-tracing model can be found in the Supporting Information.

1. Debije, M. G. \& Verbunt, P. P. C. Thirty years of luminescent solar concentrator research: solar energy for the built environment. Adv. Energy Mater. 2, 12-35, doi:DOI 10.1002/aenm.201100554 (2012).

2. Haines, C., Chen, M. \& Ghiggino, K. P. The effect of perylene diimide aggregation on the light collection efficiency of luminescent concentrators. Sol. Energy Mater. Sol. Cells 105, 287-292, doi:10.1016/j.solmat.2012.06.030 (2012).

3. Bronstein, N. D. et al. Luminescent Solar Concentration with Semiconductor Nanorods and Transfer-Printed Micro-Silicon Solar Cells. ACS Nano 8, 44-53, doi:10.1021/nn404418h (2013)

4. Purcell-Milton, F. \& Gun'ko, Y. K. Quantum dots for luminescent solar concentrators. J. Mater. Chem. 22, 16687-16697, doi:10.1039/C2JM32366D (2012)

5. Zhao, Y. \& Lunt, R. R. Transparent luminescent solar concentrators for largearea solar windows enabled by massive stokes-shift nanocluster phosphors. Adv. Energy Mater. 3, 1143-1148, doi:10.1002/aenm.201300173 (2013).

6. Currie, M. J., Mapel, J. K., Heidel, T. D., Goffri, S. \& Baldo, M. A. High-efficiency organic solar concentrators for photovoltaics. Science 321, 226-228, doi:10.1126/ science.1158342 (2008)

7. Ziessel, R., Ulrich, G., Haefele, A. \& Harriman, A. An artificial light-harvesting array constructed from multiple Bodipy dyes. J. Am. Chem. Soc. 135 11330-11344, doi:10.1021/ja4049306 (2013).

8. Bozdemir, O. A., Erbas-Cakmak, S., Ekiz, O. O., Dana, A. \& Akkaya, E. U. Towards unimolecular luminescent solar concentrators: bodipy-based dendritic energytransfer cascade with panchromatic absorption and monochromatized emission. Angew. Chem. Int. Ed. 50, 10907-10912, doi:10.1002/anie.201104846 (2011).

9. Menendez-Velazquez, A. et al. Light-recycling within electronic displays using deep red and near infrared photoluminescent polarizers. Energy Environ. Sci. 6, 72-75, doi:10.1039/C2EE23265K (2013).

10. Ding, D., Li, K., Liu, B. \& Tang, B. Z. Bioprobes based on AIE fluorogens. Acc. Chem. Res., doi:10.1021/ar3003464 (2013). phenomenon, mechanism and applications. Chem. Commun., 4332-4353, doi:10.1039/B904665H (2009).

12. Luo, J. et al. Aggregation-induced emission of 1-methyl-1,2,3,4,5 pentaphenylsilole. Chem. Commun., 1740-1741, doi:10.1039/B105159H (2001).

13. Hong, Y., Lam, J. W. \& Tang, B. Z. Aggregation-induced emission. Chem. Soc. Rev. 40, 5361-5388, doi:10.1039/c1cs15113d (2011).

14. Leow, S. W. et al. Analyzing luminescent solar concentrators with front-facing photovoltaic cells using weighted Monte Carlo ray tracing. J. Appl. Phys. 113, 214510-214519, doi:10.1063/1.4807413 (2013).

15. Sholin, V., Olson, J. D. \& Carter, S. A. Semiconducting polymers and quantum dots in luminescent solar concentrators for solar energy harvesting. J. Appl. Phys. 101, 123114, doi:10.1063/1.2748350 (2007).

16. Slooff, L. H. et al. A luminescent solar concentrator with $7.1 \%$ power conversion efficiency. Phys. Status Solidi RRL 2, 257-259, doi:10.1002/pssr.200802186 (2008).

17. Chen, S., Qin, W., Zhao, Z., Tang, B. Z. \& Kwok, H.-S. One-step fabrication of organic nanoparticles as scattering media for extracting substrate waveguide light from organic light-emitting diodes. J. Mater. Chem. 22, 13386-13390, doi:10.1039/C2JM32273K (2012).

18. Zhou, J. et al. From tetraphenylethene to tetranaphthylethene: structural evolution in AIE luminogen continues. Chem. Commun. 49, 2491-2493, doi:10.1039/C3CC00010A (2013).

19. Zhao, Z. et al. Pyrene-substituted ethenes: aggregation-enhanced excimer emission and highly efficient electroluminescence. J. Mater. Chem. 21, 7210-7216, doi:10.1039/C0JM04449K (2011).

20. Wilson, L. R. et al. Characterization and reduction of reabsorption losses in luminescent solar concentrators. Appl. Opt. 49, 1651-1661, doi:10.1364/ AO.49.001651 (2010)

21. Ross, D., Klampaftis, E., Fritsche, J., Bauer, M. \& Richards, B. S. Increased shortcircuit current density of production line CdTe mini-module through luminescent down-shifting. Sol. Energy Mater. Sol. Cells 103, 11-16, doi: 10.1016 j.solmat.2012.04.009 (2012)

22. Griffini, G. et al. Anthracene/tetracene cocrystals as novel fluorophores in thinfilm luminescent solar concentrators. RSC Advances 4, 9893-9897, doi:10.1039/ C3RA46810K (2014).

23. Porrès, L. et al. Absolute measurements of photoluminescence quantum yields of solutions using an integrating sphere. J. Fluoresc. 16, 267-273, doi:10.1007/ s10895-005-0054-8 (2006).

24. Mulder, C. L. et al. Dye alignment in luminescent solar concentrators: I. Vertical alignment for improved waveguide coupling. Opt. Express 18, A79-A90 (2010).

\section{Acknowledgments}

We thank Marc Baldo and Noel Giebink for useful discussions. This work was made possible by support from the Australian Renewable Energy Agency which funds the fellowship for WWHW and project grants within the Australian Centre for Advanced Photovoltaics. Responsibility for the views, information or advice expressed herein is not accepted by the Australian Government. JLB would like to acknowledge the support of the Australian government through an Australian Postgraduate Award.

\section{Author contributions}

J.L.B. did the experiments and calculations for the Monte Carlo simulations. J.M.W. obtained crystal structure data and solved the structures. K.P.G. and W.W.H.W. conceived the project.

\section{Additional information}

Supplementary information accompanies this paper at http://www.nature.com/ scientificreports

Competing financial interests: The authors declare no competing financial interests.

How to cite this article: Banal, J.L., White, J.M., Ghiggino, K.P. \& Wong, W.W.H. Concentrating Aggregation-Induced Fluorescence in Planar Waveguides: A Proof-of-Principle. Sci. Rep. 4, 4635; DOI:10.1038/srep04635 (2014).

This work is licensed under a Creative Commons Attribution-NonCommercialNoDerivs 3.0 Unported License. The images in this article are included in the article's Creative Commons license, unless indicated otherwise in the image credit; if the image is not included under the Creative Commons license, users will need to obtain permission from the license holder in order to reproduce the image. To view a copy of this license, visit http://creativecommons.org/licenses/by-nc-nd/3.0/ 